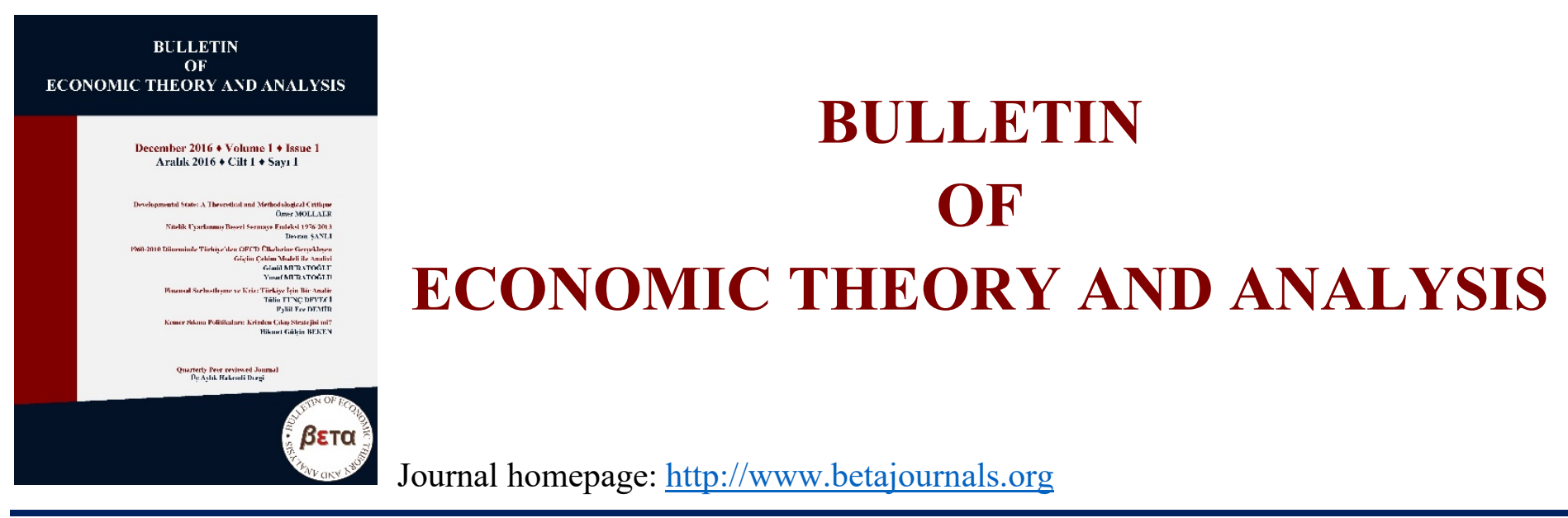

\title{
Use of Trimean in Theil-Sen Regression Analysis
}

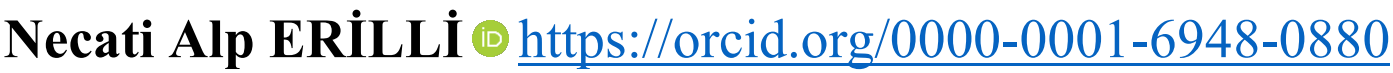

To cite this article: Erilli, A., N., (2021). Use of Trimean in Theil-Sen Regression Analysis. Bulletin of Economic Theory and Analysis, 6(1), 15-26.

Received: 11 Nov 2020

Accepted: 19 Jan 2021

Published online: 30 Jun 2021

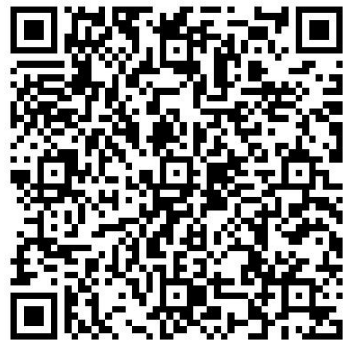




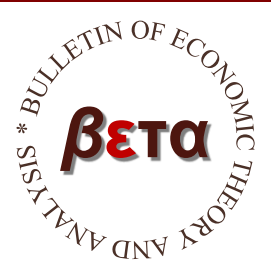

\title{
Bulletin of Economic Theory and Analysis
}

\author{
Volume VI, Issue 1, pp. 15-26, 2021
}

http://www.betajournals.org

Original Article / Araştırma Makalesi

Received / Alınma: 17.11.2020 Accepted / Kabul: 19.01.2021

\section{Use of Trimean in Theil-Sen Regression Analysis}

\author{
Necati Alp ERILLII
}

\begin{abstract}
a Assoc.. Prof. Dr., Sivas Cumhuriyet University, FEAS, Department of Econometrics, Sivas, TURKEY
(c) https://orcid.org/0000-0001-6948-0880
\end{abstract}

\begin{abstract}
Theil-Sen regression analysis is the most preferred method in non-parametric regression analysis. In the Theil-Sen method, calculations are made with the median parameter. In this study, it was proposed to calculate the trimean parameter instead of the median parameter. In this way, the effects of the outliers in the data on the model are fully reflected. In applications of one real-life and two simulation data, the results obtained with the use of trimean were more successful. It is recommended to use the trimean parameter instead of the median parameter in data structures with an excess of outliers.
\end{abstract}

Keywords

Theil-Sen

Regression, Trimean, Non-Parametric

Regression, MAPE

JEL Classification C53, C14

CONTACT Necati Alp ERILLI $₫$ aerilli@cumhuriyet.edu.tr $\equiv$ Sivas Cumhuriyet University, FEAS, Department of Econometrics, Sivas, TURKEY

\section{Introduction}

Statistical estimation studies refer to the use of statistics based on historical data to predict what may happen in the future. The most used estimation method is regression analysis. Regression analysis is a statistical technique in which we use the observed data to correlate a variable called a dependent variable and one or more independent variables. The aim is to create a regression model or estimation equation that can be used to define, predict, and control the dependent variable based on independent variables (Gujarati, 2002). Many assumptions are required to obtain successful 
estimates by regression analysis. In applications, it is not easy to provide some of these assumptions. In cases where assumptions cannot be provided, it is recommended to use flexible but less powerful non-parametric methods.

Non-parametric regression analysis can also be defined as one of the alternative estimation methods. There are a small number of non-parametric regression analysis methods in the literature. The best known and used of these is the Theil-Sen method. This method was first proposed by Theil (1950) and the procedure is firstly known as Theil's Method. After Sen (1968) highlighted the relationship to Kendall's tau it is named as the Theil-Kendall or Theil-Sen method. Theil proposed estimating the slope of a regression line as the median of the slopes of all tines joining pairs of points with different $x$ values (Theil, 1950). For a pair $\left(x_{i}, y_{i}\right)$ and $\left(x_{j}, y_{j}\right)$ the appropriate slope is $S_{i j}=\frac{\left(y_{j}-y_{i}\right)}{\left(x_{j}-x_{i}\right)}$. There will be $\frac{n(n+1)}{2}$ slopes for any data. The $\hat{\beta}_{1}$ statistic, which is the estimator of the parameter $\beta_{1}$ in simple regression analysis, is calculated as the median of the slope values: $\hat{\beta}_{1}=\operatorname{Median}\left(S_{i j}\right)$. Theil suggested for the estimation of the intercept as $\hat{\beta}_{0}=\operatorname{Median}\left(y_{i}\right)-\hat{\beta}_{1} \operatorname{Median}\left(x_{i}\right)$ (Theil, 1950; Sprent, 1989). In the Theil-Sen method, alternative methods for intercept parameter computation are also introduced, although the intercept parameter is calculated as given above. Some alternative calculations have been proposed in comparison to Theil's idea of finding the intercept parameter. Let us define $d_{i}=y_{i}-\hat{\beta}_{1} x_{i}$ calculated for all observations where $\hat{\beta}_{1}$ is calculated with the Theil-Sen method. Hodges-Lehmann method for $\hat{\beta}_{0}$ is defined as the mean value of $d_{i}\left(\hat{\beta}_{0}=\operatorname{Mean}\left(d_{i}\right)\right)$ and the optimum method for $\hat{\beta}_{0}$ which is defined as the median value of $d_{i}\left(\hat{\beta}_{0}=\operatorname{Median}\left(d_{i}\right)\right)$ ( Hodges and Lehmann, 1963). The optimum approach does not require the assumption of symmetrically distributed $d_{i}$. It is better suited especially for data with outliers. On the other hand, the Hodges-Lehmann method may not be available for data with outliers (Lehmann and Dabrera, 1975; Erilli and Alakuş, 2016).

There are many papers studied with the Theil-Sen method in the literature (Akritas et al., 1995; Fernandes and Leblanc, 2005; Lavagnini et al., 2011; Hanxiang et al., 2008; Adichie, 1967; 
Wang, 2005; Dang et al., 2008; Wilcox 1998). All of these have been studied on classical TheilSen estimates using the median parameter.

The study consists of five sections, including introduction and conclusion parts. In the second part, the trimean parameter is briefly introduced and expressed by the formula. In the third section, the proposed regression method using the trimean parameter and the significance test of the slope parameter are introduced. The strength of the proposed method in Chapter Four is compared on Theil regression method obtained with both median and trimean parameters. MAE and MAPE methods were used in comparisons and the results were evaluated. The study was completed with a conclusion section containing general assessments.

\section{Trimean Parameter}

A trimean is a number that represents the general tendency of a set of numbers or data set. Like the mean, median, and mode, it is a measure of central tendency. The trimean (TM) is a measure of a probability distribution's location defined as a weighted average of the distribution's median and its two quartiles:

$$
T M=\frac{Q_{1}+(2 \times \text { Median })+Q_{3}}{4}
$$

After Tukey has given this formula's name with a set of techniques in his book it is sometimes called Tukey's Trimean (Tukey, 1977). It is considered 'resistant' or 'robust' because it is not very affected by outliers.

\section{Trimean with Theil-Sen Regression}

With this study, it is proposed to use trimean instead of the median parameter in the calculation of both the slope parameter and the intercept parameter in the Theil-Sen regression method. The slope parameter is calculated by using trimean instead of the median of slope values calculated from dependent and independent variable binaries in the Theil-Sen method. Similarly,

the intercept parameter was also found by calculating the trimean of $d_{i}$ values: $\hat{\beta}_{0}=\operatorname{Trimean}\left(d_{i}\right)$.

\subsection{Test of Significance of Slope Parameter}

To test $\beta_{1}=0$, we can use the test statistics given in Equation.3.1 and 3.2: 


$$
|t|=\frac{|U|}{S D(U)}
$$

where

$$
U=\sum\left[\operatorname{rank}\left(y_{i}\right)-\frac{n+1}{2}\right] x_{i} \text { and } S D(U)=\sqrt{\frac{n(n+1)}{12} \sum\left(x_{i}-\bar{x}\right)^{2}}
$$

The approximate $p$-value of the test is calculated to be $\operatorname{Prob}[|Z| \geq|t|]$, where $Z$ is a random variable having a standard normal distribution (Birkes and Dodge, 1993:119).

\section{Application}

In the application part Theil-Sen regression is performed with Trimean and Median parameters separately. The proposed method is tested in 1 real-time data and 2 simulation data sets where the outliers were added by $10 \%$ to $40 \%$ to the real-time data. MAE (Mean Absolute Error) and MAPE (Mean Absolute Percentage Error) values were examined to test the validity of the results.

$$
\begin{gathered}
M A E=\frac{1}{n} \sum_{j=1}^{n}\left|y_{i}-\hat{y}_{j}\right| \\
M A P E=\left(\frac{1}{n} \sum \frac{\mid \text { Actual }- \text { Forecast } \mid}{\mid \text { Actual } \mid}\right) \times 100
\end{gathered}
$$

MAE is more robust to outliers since it does not make use of square and MAPE is asymmetric and reports higher errors if the forecast is more than the actual and lower errors when the forecast is less than the actual.

The first data set is Blood Pressure data and given in Table.1 which has 30 samples and taken from Spath (1992: 304). Sample data consist of age (independent variable) and systolic blood pressure (dependent variable) values for 30 individuals aged 17 to 69 years.

Table 1

Data Set.1

\begin{tabular}{llllllllllllllll}
\hline Variables & \multicolumn{11}{c}{ Data } \\
\hline Y (Blood Pressure) & 144 & 220 & 138 & 145 & 162 & 142 & 170 & 124 & 158 & 154 & 162 & 150 & 140 & 110 & 128 \\
X (Age) & 39 & 47 & 45 & 47 & 65 & 46 & 67 & 42 & 67 & 56 & 64 & 56 & 59 & 34 & 42 \\
Y (Blood Pressure) & 130 & 135 & 114 & 116 & 124 & 136 & 142 & 120 & 120 & 160 & 158 & 144 & 130 & 125 & 175 \\
X (Age) & 48 & 45 & 17 & 20 & 19 & 36 & 50 & 39 & 21 & 44 & 53 & 63 & 29 & 25 & 69 \\
\hline
\end{tabular}


The scatterplot of the variables is also given in Figure.1.

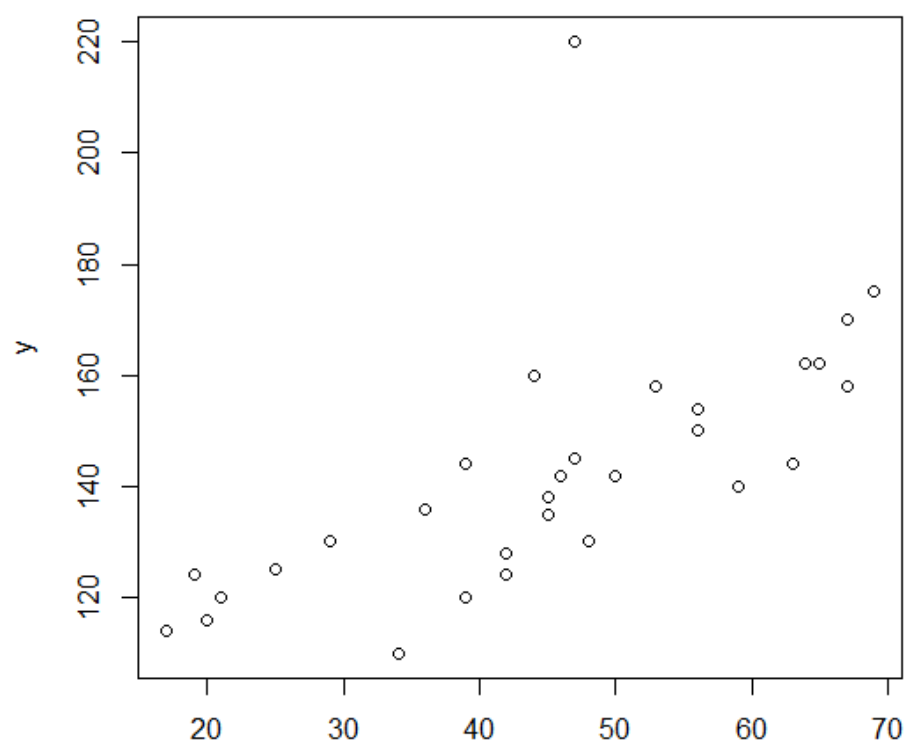

Figure 1. Scatterplot for Data.1

Parameter estimates were obtained for 9 different models using median and trimean. MAE and MAPE values were compared for 4 models where the slope parameter is estimated with median and 5 models where the slope parameter is estimated with trimean. In Table 2, the results of the estimates obtained for the original version of the data are given.

Table 2

Parameter Estimations and MAE-MAPE Results for Blood Pressure Data

\begin{tabular}{l|l|cccc}
\hline \multirow{2}{*}{$\boldsymbol{\beta}_{0}$ Calculation } & \multicolumn{4}{|c}{ Original Data } \\
\cline { 3 - 6 }$\beta_{1}$ calculation with median & $\boldsymbol{\beta}_{0}$ & $\boldsymbol{\beta}_{1}$ & MAE & MAPE \\
\hline & Theil-Sen (Median) & 64,12 & 1,36 & 50,57466667 & 305,2356789 \\
& $\mathrm{~d}_{\mathrm{i}}$ (Mean) & 31,652 & 1,36 & 56,7536 & 222,7109698 \\
& $\mathrm{~d}_{\mathrm{i}}$ (Median) & 65,8 & 1,36 & 50,53466667 & 309,7311069 \\
& $\mathrm{~d}_{\mathrm{i}}$ (Trimean) & 45,68375 & 1,36 & 53,94725 & 258,2766554 \\
\hline \multirow{5}{*}{$\beta_{1}$ calculation with trimean } & Theil-Sen (Median) & 37,1528241 & 1,952685185 & 49,09053704 & 291,1776195 \\
& Theil-Sen (Trimean) & 13,0485243 & 1,952685185 & 52,21621489 & 228,9357882 \\
& $\mathrm{~d}_{\mathrm{i}}$ (Mean) & 4,90214198 & 1,952685185 & 53,84549136 & 208,2874961 \\
& $\mathrm{~d}_{\mathrm{i}}$ (Median) & 35,673287 & 1,952685185 & 49,02344444 & 287,1833844 \\
& $\mathrm{~d}_{\mathrm{i}}$ (Trimean) & 18,7998032 & 1,952685185 & 51,0659591 & 243,5133126 \\
\hline
\end{tabular}


As for the results given in Table.2, we can clearly say that $\beta_{1}$ calculation with trimean has the best scores for both MAPE and MAE. It is found $\beta_{0}$ calculation with $\mathrm{d}_{\mathrm{i}}$ median has minimum MAE and $\beta_{0}$ calculation with $\mathrm{d}_{\mathrm{i}}$ mean has minimum MAPE.

Secondly, the above calculations were repeated by creating $10 \%, 20 \%, 30 \%$, and $40 \%$ outliers for the original data. The aim is to investigate the effect of the proposed method on deviating values in the data. Figure. 2 shows the scatterplot for the data with $10 \%, 20 \%, 30 \%$, and $40 \%$ outliers.
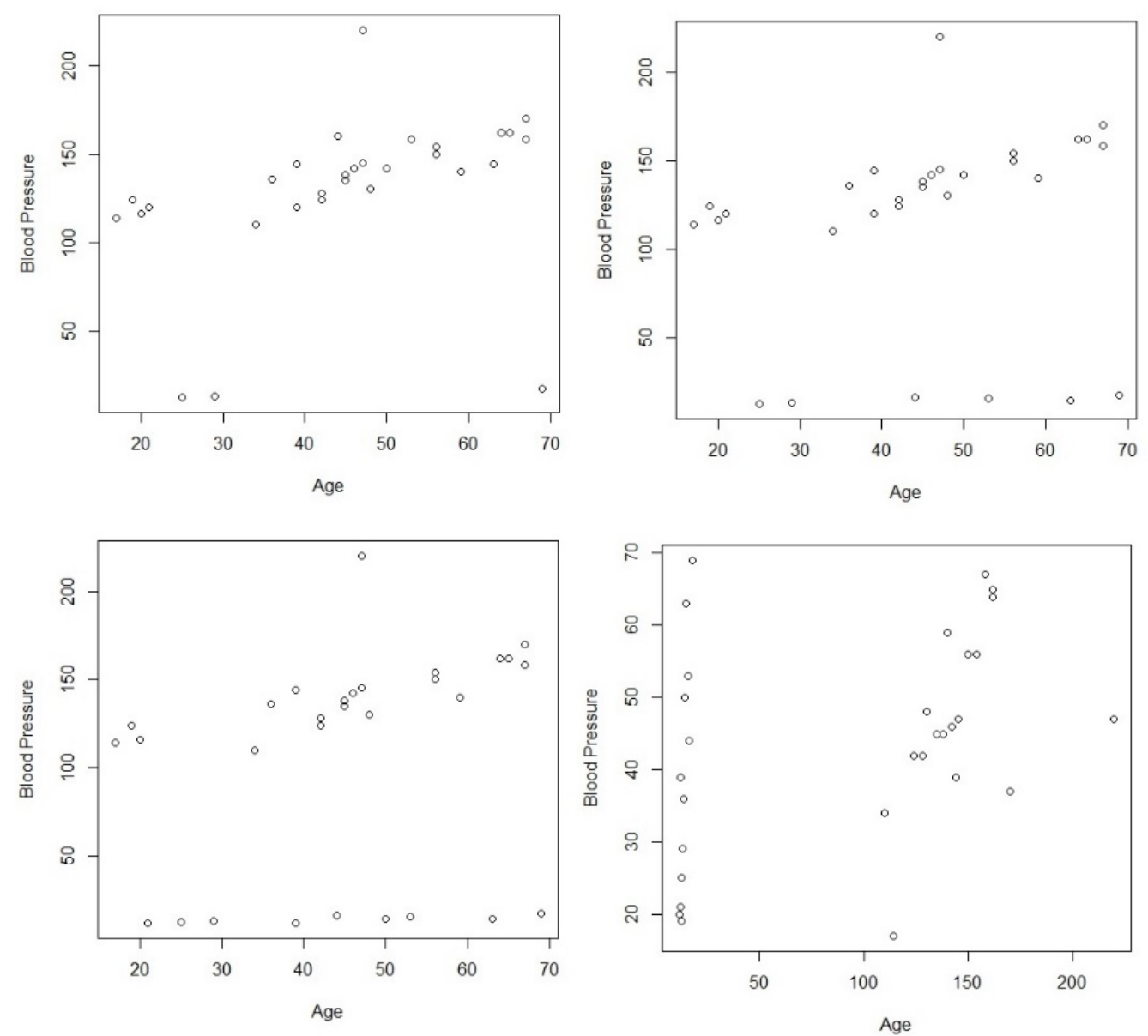

Figure 2. Scatterplot with 10\%, 20\%, 30\%, and 40\% outliers for Blood Pressure Data

In Tables 3,4,5 and 6, the results of the Blood Pressure data with outliers added are given. When we look at the results in Table.3, it is seen that the best model is the calculation with trimean according to MAPE and the calculation with median according to MAE. 
Table 3

Parameter Estimations and MAE-MAPE Results for Blood Pressure Data with 10\% outliers

\begin{tabular}{l|l|cccc}
\hline \multirow{2}{*}{$\boldsymbol{\beta}_{0}$ Calculation } & \multicolumn{4}{|c}{ Blood Pressure Data with 10\% Outlier } \\
\cline { 3 - 6 }$\beta_{1}$ calculation with median & $\boldsymbol{\beta}_{0}$ & $\boldsymbol{\beta}_{1}$ & MAE & MAPE \\
\hline & Theil-Sen (Median) & 93,5 & 1 & 21,26666667 & 90,01196426 \\
& $\mathrm{~d}_{\mathrm{i}}$ (Mean) & 84,5 & 1 & 23,83333333 & 85,79972089 \\
& $\mathrm{~d}_{\mathrm{i}}$ (Median) & 95 & 1 & 21,23333333 & 90,98957131 \\
& $\mathrm{~d}_{\mathrm{i}}$ (Trimean) & 92,6875 & 1 & 21,34166667 & 89,5215978 \\
\hline \multirow{5}{*}{$\beta_{1}$ calculation with trimean } & Theil-Sen (Median) & 86,1749226 & 1,160990712 & 21,61971104 & 89,56132911 \\
& Theil-Sen (Trimean) & 84,6669892 & 1,160990712 & 21,71344169 & 88,62290537 \\
& $\mathrm{~d}_{\mathrm{i}}$ (Mean) & 77,2339525 & 1,160990712 & 23,85265222 & 85,17595114 \\
& $\mathrm{~d}_{\mathrm{i}}$ (Median) & 86,1455108 & 1,160990712 & 21,61971104 & 89,54172461 \\
& $\mathrm{~d}_{\mathrm{i}}$ (Trimean) & 85,2474845 & 1,160990712 & 21,65357327 & 88,96768046 \\
\hline
\end{tabular}

As for the results given in Table.4, it is clearly said that $\beta_{1}$ calculation with trimean has the best scores for both MAPE and MAE. It is found $\beta_{0}$ calculation with $\mathrm{d}_{\mathrm{i}}$ median has minimum MAE and $\beta_{0}$ calculation with $\mathrm{d}_{\mathrm{i}}$ mean has minimum MAPE just like results given in Table.2.

Table 4

Parameter Estimations and MAE-MAPE Results for Blood Pressure Data with 10\% outliers

\begin{tabular}{l|l|cccc}
\hline \multirow{2}{*}{$\boldsymbol{\beta}_{0}$ Calculation } & \multicolumn{4}{|c}{ Blood Pressure Data with 20\% Outlier } \\
\cline { 3 - 6 }$\beta_{1}$ calculation with median & $\boldsymbol{\beta}_{0}$ & $\boldsymbol{\beta}_{1}$ & MAE & MAPE \\
\hline & Theil-Sen (Median) & 89,6764706 & 0,941176471 & 33,60117647 & 167,1870134 \\
& $\mathrm{~d}_{\mathrm{i}}$ (Mean) & 73,294902 & 0,941176471 & 41,12815686 & 152,4432758 \\
& $\mathrm{~d}_{\mathrm{i}}$ (Median) & 95,2941176 & 0,941176471 & 32,70117647 & 173,5558044 \\
& $\mathrm{~d}_{\mathrm{i}}$ (Trimean) & 93,7463235 & 0,941176471 & 32,8604902 & 171,7378408 \\
\hline \multirow{3}{*}{$\beta_{1}$ calculation with trimean } & Theil-Sen (Median) & 91,3715686 & 0,903921569 & 33,58503268 & 167,1555246 \\
& Theil-Sen (Trimean) & 89,5386029 & 0,903921569 & 33,98473856 & 165,1562573 \\
& $\mathrm{~d}_{\mathrm{i}}$ (Mean) & 74,9763399 & 0,903921569 & 41,09785621 & 152,3765839 \\
& $\mathrm{~d}_{\mathrm{i}}$ (Median) & 97,3803922 & 0,903921569 & 32,62928105 & 173,9750902 \\
& $\mathrm{~d}_{\mathrm{i}}$ (Trimean) & 95,7231618 & 0,903921569 & 32,80802288 & 172,0324633 \\
\hline
\end{tabular}

Results in Table.5 shows that $\beta_{1}$ calculation with trimean has the best scores for both MAPE and MAE. It is found $\beta_{0}$ calculation with Theil-Sen Trimean has minimum MAPE and $\beta_{0}$ calculation with $\mathrm{d}_{\mathrm{i}}$ median has minimum MAE. 
Table 5

Parameter Estimations and MAE-MAPE Results for Blood Pressure Data with 30\% outliers

\begin{tabular}{l|l|cccc}
\hline \multirow{2}{*}{$\boldsymbol{\beta}_{0}$ Calculation } & \multicolumn{4}{|c}{ Blood Pressure Data with 30\% Outlier } \\
\cline { 3 - 6 }$\beta_{1}$ calculation with median & $\boldsymbol{\beta}_{0}$ & $\boldsymbol{\beta}_{1}$ & MAE & MAPE \\
\hline & Theil-Sen (Median) & 87 & 0,923076923 & 44,40461538 & 247,7264811 \\
& $\mathrm{~d}_{\mathrm{i}}$ (Mean) & 62,6517949 & 0,923076923 & 53,28235897 & 206,7326515 \\
& $\mathrm{~d}_{\mathrm{i}}$ (Median) & 94,8076923 & 0,923076923 & 43,82512821 & 262,6567125 \\
& $\mathrm{~d}_{\mathrm{i}}$ (Trimean) & 70,4350962 & 0,923076923 & 50,16903846 & 219,6004656 \\
\hline \multirow{5}{*}{$\beta_{1}$ calculation with trimean } & Theil-Sen (Median) & 80,8444444 & 1,058363858 & 44,31442409 & 246,790076 \\
& Theil-Sen (Trimean) & 56,1626603 & 1,058363858 & 53,31658018 & 205,2601872 \\
& $\mathrm{~d}_{\mathrm{i}}$ (Mean) & 56,5458445 & 1,058363858 & 53,16330647 & 205,8936901 \\
& $\mathrm{~d}_{\mathrm{i}}$ (Median) & 87,2316239 & 1,058363858 & 43,71779406 & 258,9097462 \\
& $\mathrm{~d}_{\mathrm{i}}$ (Trimean) & 64,0612408 & 1,058363858 & 50,15714794 & 218,3185878 \\
\hline
\end{tabular}

Table. 6 results Show that $\beta_{1}$ calculation with trimean has the best scores for both MAPE and MAE. It is found $\beta_{0}$ calculation with $\mathrm{d}_{\mathrm{i}}$ median has minimum MAE and $\beta_{0}$ calculation with $\mathrm{d}_{\mathrm{i}}$ mean has minimum MAPE just like results given in Table. 2 and Table.4.

Table 6

Parameter Estimations and MAE-MAPE Results for Blood Pressure Data with 40\% outliers

\begin{tabular}{l|l|cccc}
\hline \multirow{2}{*}{$\boldsymbol{\beta}_{0}$ Calculation } & \multicolumn{4}{|c}{ Blood Pressure Data with 20\% Outlier } \\
\cline { 2 - 6 }$\beta_{1}$ calculation with median & $\boldsymbol{\beta}_{0}$ & $\boldsymbol{\beta}_{1}$ & MAE & MAPE \\
\hline & Theil-Sen (Median) & 64,12 & 1,36 & 50,57466667 & 305,2356789 \\
& $\mathrm{~d}_{\mathrm{i}}$ (Mean) & 31,652 & 1,36 & 56,7536 & 222,7109698 \\
& $\mathrm{~d}_{\mathrm{i}}$ (Median) & 65,8 & 1,36 & 50,53466667 & 309,7311069 \\
& $\mathrm{~d}_{\mathrm{i}}$ (Trimean) & 45,68375 & 1,36 & 53,94725 & 258,2766554 \\
\hline \multirow{5}{*}{$\beta_{1}$ calculation with trimean } & Theil-Sen (Median) & 37,1528241 & 1,952685185 & 49,09053704 & 291,1776195 \\
& Theil-Sen (Trimean) & 13,0485243 & 1,952685185 & 52,21621489 & 228,9357882 \\
& $\mathrm{~d}_{\mathrm{i}}$ (Mean) & 4,90214198 & 1,952685185 & 53,84549136 & 208,2874961 \\
& $\mathrm{~d}_{\mathrm{i}}$ (Median) & 35,673287 & 1,952685185 & 49,02344444 & 287,1833844 \\
& $\mathrm{~d}_{\mathrm{i}}$ (Trimean) & 18,7998032 & 1,952685185 & 51,0659591 & 243,5133126 \\
\hline
\end{tabular}

Model significance was also calculated for the models which parameter estimates given above. Thus, $\beta_{1}$ is found significant at 0,05 and 0,01 level with values of $|t|=\frac{|U|}{S D(U)}=\frac{3213}{75,0646}=4,4313$.

Thirdly, the proposed method was applied on two simulation data, one with 14 observations and one with 7 observations. In Figure. 3 it is given scatterplot Simulation-1 and Simulation-2 Data. 

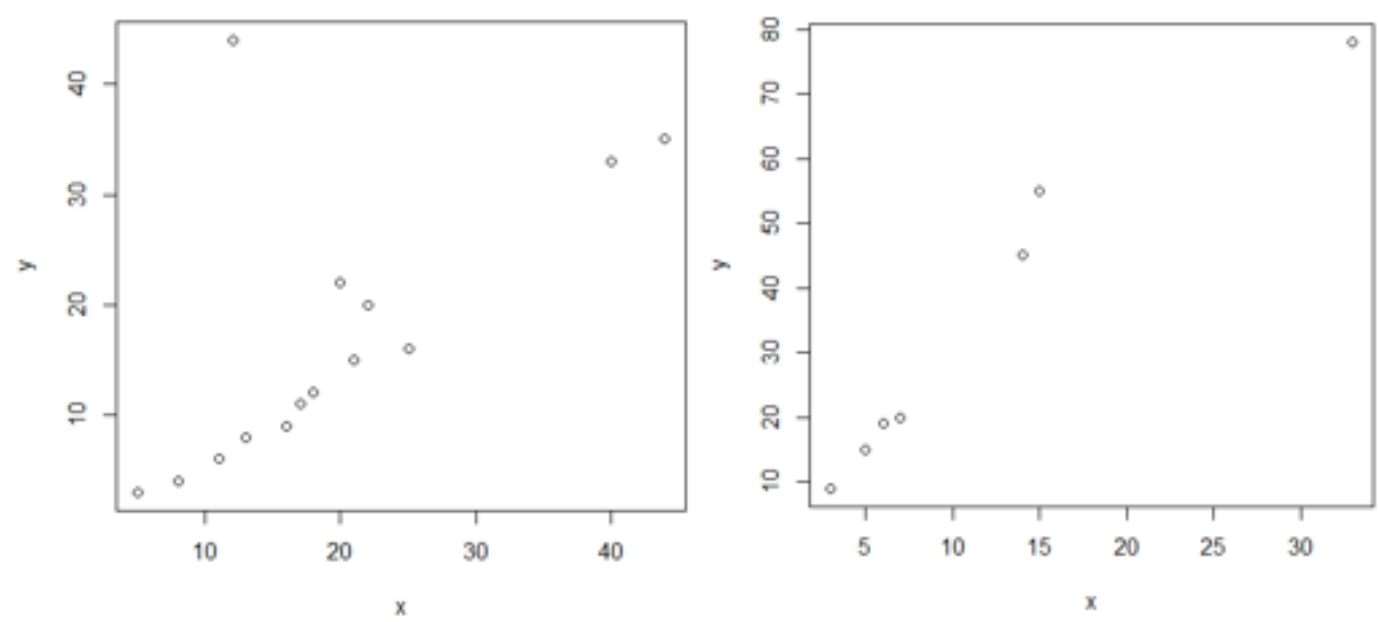

Figure 3. Scatterplot of Simulation-1 and Simulation-2 Data

Results of simulation data are given in Table.7 and Table.8 separately. For the first simulation data, the best MAE value was obtained by $\beta_{1}$ calculation with median, while the best MAPE value was obtained by $\beta_{1}$ calculation with trimean given in Table.7.

Table 7

Parameter Estimations and MAE-MAPE Results for Simulation Data-1

\begin{tabular}{l|l|cccc}
\hline & \multirow{2}{*}{$\boldsymbol{\beta}_{0}$ Calculation } & \multicolumn{4}{c}{ Simulation Data-1 } \\
\cline { 3 - 6 } & & $\boldsymbol{\beta}_{0}$ & $\boldsymbol{\beta}_{1}$ & MAE & MAPE \\
\hline \multirow{3}{*}{$\beta_{1}$ calculation with median } & Theil-Sen (Median) & $-1,3076923$ & 0,846153846 & 4,527472527 & 23,18496979 \\
& $\mathrm{~d}_{\mathrm{i}}$ (Mean) & -2 & 0,846153846 & 4,32967033 & 20,69790329 \\
& $\mathrm{~d}_{\mathrm{i}}$ (Median) & $-2,7692308$ & 0,846153846 & 4,186813187 & 18,15427628 \\
& $\mathrm{~d}_{\mathrm{i}}$ (Trimean) & $-2,5192308$ & 0,846153846 & 4,222527473 & 18,95034281 \\
\hline \multirow{3}{*}{$\beta_{1}$ calculation with trimean } & Theil-Sen (Median) & $-0,8413462$ & 0,819505495 & 4,556456044 & 24,30225605 \\
& Theil-Sen (Trimean) & 0,05103022 & 0,819505495 & 4,938903061 & 31,75746647 \\
& $\mathrm{~d}_{\mathrm{i}}$ (Mean) & $-1,467033$ & 0,819505495 & 4,399489796 & 21,00146286 \\
& $\mathrm{~d}_{\mathrm{i}}$ (Median) & $-2,556044$ & 0,819505495 & 4,293406593 & 17,86369402 \\
& $\mathrm{~d}_{\mathrm{i}}$ (Trimean) & $-2,2754808$ & 0,819505495 & 4,293406593 & 18,48987884 \\
\hline
\end{tabular}

In Table. 8 , it is seen that the calculation of $\beta_{1}$ with trimean results has minimum MAE and MAPE. 
Table 8

Parameter Estimations and MAE-MAPE Results for Simulation Data-2

\begin{tabular}{l|l|cccc}
\hline \multirow{2}{*}{$\boldsymbol{\beta}_{0}$ Calculation } & \multicolumn{4}{|c}{ Simulation Data-2 } \\
\cline { 3 - 6 }$\beta_{1}$ calculation with median & $\boldsymbol{\beta}_{0}$ & $\boldsymbol{\beta}_{1}$ & MAE & MAPE \\
\hline & Theil-Sen (Median) & $-2,75$ & 3,25 & 3,107142857 & 7,100165916 \\
& $\mathrm{~d}_{\mathrm{i}}$ (Mean) & $-4,1071429$ & 3,25 & 3,591836735 & 10,08581122 \\
& $\mathrm{~d}_{\mathrm{i}}$ (Median) & $-0,75$ & 3,25 & 2,75 & 4,605029473 \\
& $\mathrm{~d}_{\mathrm{i}}$ (Trimean) & -1 & 3,25 & 2,767857143 & 4,7383501 \\
\hline \multirow{5}{*}{$\beta_{1}$ calculation with trimean } & Theil-Sen (Median) & $-2,0208333$ & 3,145833333 & 2,973214286 & 6,607061311 \\
& Theil-Sen (Trimean) & 0,01041667 & 3,145833333 & 2,707589286 & 5,004651325 \\
& $\mathrm{~d}_{\mathrm{i}}$ (Mean) & $-2,8720238$ & 3,145833333 & 3,277210884 & 8,479637096 \\
& $\mathrm{~d}_{\mathrm{i}}$ (Median) & $-0,4375$ & 3,145833333 & 2,675595238 & 4,532538611 \\
& $\mathrm{~d}_{\mathrm{i}}$ (Trimean) & $-0,4270833$ & 3,145833333 & 2,676339286 & 4,543517977 \\
\hline
\end{tabular}

When we look at the model significances; $\beta_{1}$ is found significant at 0,05 and 0,01 level with values of $|t|=\frac{|U|}{S D(U)}=\frac{563}{166.988}=3.3713$ in Simulation Data-1 and $\beta_{1}$ is found significant at 0,05 level with values of $|t|=\frac{|U|}{S D(U)}=\frac{118}{54.85}=2.151$ in Simulation Data-2.

\section{Conclusion}

In this study, it was proposed to use the trimean parameter instead of the median parameter in Theil-Sen regression analysis. Thus, the contribution of the effect of the outliers to the model was tried to be investigated. In the proposed method, trimean was used separately for both the slope parameter and the intercept parameter. As a result of applications on one real-time data and two simulation data, model comparisons were made according to MAE and MAPE criteria. Besides, the efficiency of the method was tested by adding $10 \%, 20 \%, 30 \%$, and $40 \%$ outliers to the realtime data. The results of the analysis showed that the calculations with trimean were more successful than those with the median. The best model estimation methods can be said to be $\beta_{1}$ calculation with trimean and $\beta_{0}$ calculation with di (Mean) and di (Median).

The most common method of non-parametric regression analysis is perhaps the Theil-Sen method. In this study, the estimation results obtained by the proposed trimean parameter instead of the median parameter were successful. Finally, the use of the Trimean mean in other nonparametric statistical methods is also proposed to be investigated. 


\section{References}

Adichie, J. N. (1967). Estimates of regression parameters based on rank tests. Annals of Mathematical Statistics, 38, 894-904.

Akritas, M. G., Murphy, S. A., \& LaValley, M. P. (1995). The Theil-Sen estimator with doubly censored data and applications to astronomy. J. Amer. Statist. Assoc., 90, 170-177.

Birkes, D. \& Dodge, Y. (1993). Alternative Methods of Regression. John Wiley \& Sons Inc., NY, USA.

Dang, X., Peng, H., Wang, X. \& Zhang, H. (2008). Theil-Sen Estimators in a Multiple Linear Regression Model, Olemiss Edu.

Erilli, N. A. \& Alakuş, K. (2016). Parameter Estimation In Theil-Sen regression analysis with Jackknife method. Eurasian Econometrics, Statistics \& Empirical Economics Journal, 5, 28-41.

Fernandes, R. \& Leblanc S. G. (2005). Parametric (modified least squares) and non-parametric (Theil-Sen) linear regressions for predicting biophysical parameters in the presence of measurement errors. Remote Sensing of Environment, 95, 303-316.

Gujarati, D. N. (2002). Basic Econometrics. McGraw Hill pub., NY, USA.

Hanxiang, P., Shaoli W. \& Xueqin, W. (2008). Consistency and asymptotic distribution of the Theil-Sen estimator. Journal of Statistical Planning and Inference, 138, 1836-1850.

Hodges, J. L. \& Lehmann, E. L., (1963). Estimates of location based on rank tests. Ann. Math. Statist. 34, 598-611.

Lavagnini, I., Badocco, D., Pastore, P. \& Magno, F. (2011). Theil-Sen nonparametric regression technique on univariate calibration, inverse regression and detection limits. Talanta, 87, p.180-188.

Lehmann, E. L., \& Dabrera H. J. M. (1975). Nonparametrics: Statistical Methods Based on Ranks. SF, USA: Holden-Day Inc.

Sen, P. K. (1968). Estimates of the regression coefficient based on Kendall's tau. Journal of the American Statistical Association, 63, 1379-1389.

Spath, H. (1992). Mathematical Algorithms for Linear Regression. London: Academic Press.

Sprent, P. (1989). Applied Nonparametric Statistical Methods. Chapman and Hall Pub., London, UK.

Theil, H. (1950). A-Rank invariant method of linear and polynomial regression analysis. III. Nederl. Akad. Wetensch.Proc., Series A, 53, 1397-1412.

Tukey, J. W. (1977). Exploratory Data Analysis. Reading, M A: Addison-Wesley, 46-47. 
Wang, X. Q. (2005). Asymptotics of the Theil-Sen estimator in simple linear regression models with a random covariate. Nonparametric Statist. 17, 107-120.

Wilcox, R. R. (1998). Simulations on the Theil-Sen regression estimator with right-censored data. Statistics \& Probability Letters, 39, 43-47. 